\title{
Medicina Baseada em Evidências: a arte de aplicar o conhecimento científico na prática clínica
}

\author{
A.A. LOPES
}

Área de Concentração em E pidemiologia Clínica do Curso de Pós-Graduação em Medicina da Faculdade de Medicina da Universidade Federal da Bahia, Salvador, BA.

\begin{abstract}
RESUMO - Este artigo foi escrito com o objetivo de descrever o conceito de Medicina Baseada em Evidências (MBE) e as competências necessárias para a sua prática. MBE deve ser vista como a integração da experiência clínica com a capacidade de analisar e aplicar racionalmente a informação científica ao cuidar de pacientes. A aplicação de métodos e estratégias para fortalecer 0 alicerce científico do médico, sem desprezar os valores
\end{abstract}

\section{NTRODUÇÃO}

Um movimento denominado Medicina Baseada em Evidências (Evidence-Based Medicine, EBM ou $\mathrm{MBE})^{1}$ tem ajudado a ampliar a discussão sobre o ensino e a prática da medicina. E mbora o termo Medicina Baseada em Evidências tenha sido introduzido recentemente, os métodos didáticos que Ihe servem de alicerce não são totalmente novos ${ }^{2}$. Não se pode negar, no entanto, que a MBE, sendo um movimento voltado para a formação de médicos com espírito crítico aguçado e aptos a manter o processo de educação continuada, tem ajudado a definir novas estratégias e métodos didático-pedagógicos e a divulgar outros anteriormente desenvolvidos. Este artigo foi escrito com o objetivo de descrever o que se entende por Medicina Baseada em E vidências e as competências necessárias para os que desejam praticá-la.

O que significa Medicina Baseada em Evidências? MBE se traduz pela prática da medicina em um contexto em que a experiência clínica é integrada com a capacidade de analisar criticamente e aplicar de forma racional a informação científica de forma a melhorar a qualidade da assistência médica. Na MBE, as dúvidas que surgem ao resolver problemas de pacientes são os principais estímulos para que se procure atualizar os conhecimentos $^{1,3}$. A filosofia da MBE guarda similaridades e pode ser integrada com a metodologia de ensino-aprendizagem denominada Aprendizado Baseado em Problemas 4 . A aquisição de conhecimentos de Epidemiologia Clínica, o desenvolvi- humanitários da profissão, deverá contribuir para a melhoria da qualidade da assistência médica que é oferecida no Brasil. As Escolas e Associações Médicas podem desempenhar importantes papéis na promoção da MBE.

UNITERMOS: Medicina Baseada em Evidências. Educação médica. Aprendizado baseado em problemas.

mento do raciocínio científico, atitudes de autoaprendizagem e capacidade de integrar conhecimentos de diversas áreas são fundamentais para a prática da $\mathrm{MBE}^{3}$.

Alguém pode ser considerado possuidor das competências necessárias para a prática da MBE quando for capaz de:

1. identificar os problemas rel evantes do paciente; 2. converter os problemas em questões que conduzam às respostas necessárias;

3. pesquisar eficientemente as fontes de informação; 4. avaliar a qualidade da informação e a força da evidência, favorecendo ou negando o valor de uma determinada conduta ${ }^{5-10}$;

5. chegar a uma conclusão correta quanto ao significado da informação;

6. aplicar as conclusões dessa avaliação na mel horia dos cuidados prestados aos pacientes.

\section{Como descrever o problema no contexto da MBE?}

No contexto da MBE, o problema deve enfocar o paciente. Ao definir o problema, deve ser feito um balanço entre precisão e prolixidade (Quadro). Dependendo de cada situação, existem determinadas características do paciente que devem ser levadas em consideração na descrição do problema, de forma a facil itar a local ização das informações necessárias. Por exemplo, se a febre é uma das manifestações clínicas, o fato de o paciente ser uma criança de dois anos de idade, e não um adulto, modifica de forma importante o contexto do problema.

A construção da questão no contexto da MBE 
análise de um artigo científico visando obter resposta para uma questão específica:

- O Objetivo do Estudo

- A Metodologia Empregada

- Os Resultados

- A Aplicabilidade dos Resultados na Prática

O objetivo do estudo permite concluir se o artigo tem relação com a questão clínica. A análise da metodologia do trabalho permite avaliar a credibilidade que merece os resultados encontrados. Como a metodologia de um trabalho está intimamente ligada com o objetivo, a análise metodológica não pode ser divorciada da questão clínica. Ao se avaliar trabalhos sobre o valor de testes diagnósticos, por exemplo, deve ser observado se o resultado do teste foi definido sem um conhecimento do diagnóstico definitivo (i.e., o diagnóstico de acordo com o padrão ouro) ou vice-versa, de forma a evitar viés de observação (e.g., erros ao se classificar um teste diagnóstico como positivo ou negativo ou a doença como presente ou ausente). Quando o artigo é sobre a eficácia de intervenções preventivas ou terapêuticas, é importante que a designação (ou alocação) para os grupos seja feita de forma randômica (ou aleatória) com o objetivo de garantir probabilidades iguais de que pessoas com diferentes características façam parte de qualquer um dos grupos de tratamento.

Os conhecimentos necessários para que se analisem os resultados também dependem da natureza da questão. $\mathrm{Na}$ análise de estudos sobre testes diagnósticos, por exemplo, é necessário que se entenda pelo menos o significado dos termos sensibilidade e especificidade. $O$ entendimento do significado dos termos risco relativo (relative risk), redução do risco relativo (rel ativerisk reduction ou RRR), redução do rico absoluto (absolute risk reduction), número necessário para tratar (number needed to treat, ou NNT), sobrevida cumulativa (cumulative survival) é importante para conclusões corretas sobre estudos de terapêutica e prognóstico.

\section{Aplicação da informação científica}

No processo de praticar MBE, que vai da identificação do problema à escolha da alternativa a ser adotada, não se pode esquecer que cada pessoa que procura cuidados médicos é um ser único, apesar de possuir características similares a diversos outros pacientes. Evidências que vêm de estudos realizados com grupos de pacientes ajudam a tomar as decisões mais acertadas, mas não podem ser desvinculadas da experiência clínica. Para estabelecer as limitações em se aplicar os resultados de um determinado estudo para solucionar o problema de um paciente, as seguintes perguntas podem ajudar:

1) Se o estudo fosse realizado no local onde exerço medicina, os resultados seriam semelhantes aos encontrados pelo investigador?

2) O estudo oferece informações que permitam avaliar se os resultados dependem de características demográficas e clínicas dos pacientes, como idade, sexo, nível educacional, gravidade da condição e doenças associadas?

3) Os benefícios foram avaliados juntamente com os custos e riscos?

4) As informações real mente ajudam a orientar os meus pacientes?

5) As informações ampliam a minha capacidade de colaborar com colegas da minha área e com outros profissionais de saúde?

\section{Comentários finais}

A filosofia da MBE é condizente com a idéia de que a boa prática médica requer integração da ci ênci a e da arte. A introdução nas escol as médicas de métodos didáticos que estimulem o auto-aprendizado, bem como de cursos de E pidemiol ogia A plicada aos Problemas Clínicos e de Análise Crítica de Trabalhos Científicos, deverá contribuir para a formação de profissionais capazes de selecionar adequadamente a fonte do conhecimento e de avaliar criteriosamente como se transferir a informação para a prática médica. Além do mais, é importante que as escolas utilizem métodos didáticos que capacitem o estudante a pesquisar e criticar a informação científica, contribuindo, desta forma, para o desenvolvimento de atitudes que resultem na mel horia da qualidade dos cuidados que o mesmo venha prestar aos seus pacientes ao se tornar médico. O fato de que a escola médica onde o profissional se formou não tenha atuado mais diretamente para criar a capacidade de integrar o conhecimento científico com a experiência clínica, não deve servir de justificativa para que se despreze a prática da MBE. As Associações e as Escolas Médicas podem desempenhar importantes papéis nesse sentido, através dos seus programas de educação continuada, cursos de extensão e de pósgraduação.

\section{SUMMARY}

\section{Evidence-Based Medicine: the art of app- lying the scientific knowledge in the clinical practice}

This article was written with the objective of describing the concept of Evidence Based Medicine (EBM) and the competences required for its prac- 
tice. EBM should be viewed as an integration of clinical experience with the ability to analyze and rationally apply the scientific information while taking care of patients. The application of methods and strategies to enhance the scientific background of the physician while taking into account the humanitarian values of the medical profession should contribute to improve the quality of the medical care that is offered in Brazil. The Medical Schools and Associations may play important roles in the promotion of EBM. [Rev Ass Med Bras; 46(3): 285-8]

KEY WORDS: Evidence-based Medicine, Medical Education. Problem-based Learning.

\section{REFERÊNCIAS BIBLIOGRÁFICAS}

1. Evidence-Based Medicine Working Group.Evidence-based medicine. A new approach to teaching the practice of medicine. J AMA 1992; 268:2420-2425.

2. Lopes AA. Raciocínio Clínico e Tomada de Decisões em Medicina: um curso integrando medicina interna e epidemiologia. Rev Bras Ed Med 1991; 45: 222-224.

3. Sackett DL, Richardson WS, Rosemberg WS, Rosenberg W, Haynes BR. Evidence-Based Medicine: how to practice and teach EBM. Churchill Livingstone, 1997.

4. BlighJ . Problem-based learning in medicine: an introduction. Postgrad Med J 1995; 7: 323-326.

5. Guyatt GH, Sackett DL, Cook DJ . Users' guides to the medical literature. II. How to use an article about therapy or prevention. A. Are the results of the study valid?. J AMA 1993; 270:2598-2601.

6. Guyatt GH, Sackett DL, Cook DJ . U sers' guides tothemedical literature. II. How to use an article about therapy or prevention. $B$. What were the results and will they help me in caring for my patients? J AMA 1994; 271:59-63.

7. J aeschke R, Guyatt G, Sackett DL. Users' guides to the medical literature. III. How to usean articleabout a diagnostic test. A. Are the results of the study valid? J AMA 1994; 271:389-391.

8. J aeschke R, Guyatt G, Sackett DL. III. How to use an article about a diagnostic test. B. What are the results and will they help me in caring for my patients? J AMA 1994; 271:703-707.

9. Levine M, Walter S, Lee H, Haynes T, Holbrook A, Moyer V. IV. How to use an article about harm? J AMA 1994; 271:703-707.

10. Laupacis A, Wells G, Richardson WS, Tugwell P. Users' guides to the medical literature. V. How to use an article about prognosis. J AMA 1994; 272:234-237.

11. Fletcher HR, Fletcher SW, Wagner EH; trad. Duncan BB, Schmidt MI. Epidemiologia Clínica: Elementos Essenciais, 3 ed. Porto Alegre, RS, Artes Médicas, 1996.

12. Friedland DJ . Evidence-Based Medicine: A Framework for Clinical Practice, Stanford, CT, Appleton \& Lange, 1998.

13. Lopes AA. Statistical Inference: Basic concepts. BrasJ Infect Dis. 1998; 2: 62-9.

14. Lopes AA. Statistical Inference: The normal and related distribution. Bras J Infect Dis. 1998; 2: 170-4.

15. Lopes AA. Statistical Inference: Statistical Hypothesis Testing and Confidence Interval Estimation. Bras J Infect Dis. 1998; 2: 214-26. 